LETTER TO JMG

\title{
Genetic analysis of PSORS2 markers in a UK dataset supports the association between RAPTOR SNPs and familial psoriasis
}

\author{
F Capon, C Helms, C D Veal, D Tillman, A D Burden, J N Barker, A M Bowcock, R C Trembath
}

J Med Genet 2004;41:459-460. doi: 10.1136/jimg.2004.018226

$\mathrm{P}$ soriasis [MIM 177900] is a chronic and disfiguring skin disorder, which is inherited as a multifactorial trait. Genome wide scans have repeatedly mapped a major disease susceptibility locus (PSORS1) to the Major Histocompatibility Complex (MHC), on chromosome 6p21 (reviewed by Capon et $\mathrm{al}^{2}$ ). Outside of the MHC, at least eight additional susceptibility intervals have been reported (PSORS2-9) (reviewed by Capon et $\mathrm{al}^{2}$ ). The PSORS2 interval [MIM 602723] was originally mapped to chromosome 17q25, in a sample of extended pedigrees presenting with disease segregation across multiple generations. ${ }^{3}$ Linkage to PSORS2 was later replicated in independently ascertained cohorts. ${ }^{4-6}$ Conversely, two distinct genome wide scans carried out by our group failed to detect any evidence for linkage to PSORS2, in United Kingdom cohorts of European descent. ${ }^{7}$

High density genetic analysis of the PSORS2 interval recently identified two distinct association peaks, both defined by a small number of non-coding single nucleotide polymorphisms (SNPs). ${ }^{9}$ The proximal peak spans a $20 \mathrm{~kb}$ genomic segment where a putative susceptibility allele, mapping between the SLC9ARl and NAT9 genes, abolishes a RUNXI binding site. The less characterised distal region of association lies $6 \mathrm{Mb}$ away, within intron 3 of the RAPTOR gene [MIM *607130]. ${ }^{9}$ To define the relevance of PSORS2 genetic variation in the United Kingdom, we have analysed eight representative SNPs, selected from both association peaks. While our results do not support a pathogenic involvement of SLC9ARI/NAT9 variants, they do provide evidence for association between familial psoriasis and RAPTOR SNPs.

\section{METHODS}

Our patient cohort included a total of 233 independent parent-offspring trios of northern European origin. Of these, 116 were sampled from the family cohort previously described by Veal et al. $^{8}$ The 117 remaining trios were ascertained through an affected proband, as detailed elsewhere. ${ }^{8}$ All samples were collected following approval of the Guy's and St Thomas' hospitals ethics Committee of King's College, London. All subjects participating in this study gave their informed consent.

Based on the reported significance of disease association the following five markers were selected from the proximal association peak: SNP8 (rs7420); SNP9 (rs734232, abolishing the RUNX1 site); SNP1l (rs895691); SNP12 (rs12797); and SNP15 (rs2305214). Based on the same criterion, three further SNPs (rs1564864, rs2019154, and rs869190) were selected from the distal association peak. Following DNA extraction from blood lymphocytes, SNP genotyping was carried out by fluorescence polarisation template directed dye incorporation as described by Speckman et $\mathrm{al}^{10}$ or with Sequenom MassArray technology (Sequenom Inc). Family based association analysis was carried out using the

\section{Key points}

- Psoriasis is a multifactorial skin disorder. The major disease susceptibility locus (PSOSRS1) maps to the MHC region on chromosome 6p21. Eight additional PSORS loci have been identified outside of the MHC, with the PSORS2 interval showing linkage to psoriasis in a range of independently ascertained samples.

- High density genetic analysis of the PSORS2 locus recently identified two distinct association peaks, encompassing respectively the SLC9AR1-NAT9 genes and the third intron of the RAPTOR gene. To assess the relevance of these findings to the UK population, we have analysed eight representative single nucleotide polymorphisms (SNPs), selected from both the association peaks, in a large dataset of 233 parent-offspring trios.

- The examination of the entire dataset only provided evidence for association at RAPTOR SNP rs2019154 $(p=0.027)$. Restricting the analysis to the 116 trios with a documented family history of psoriasis increased the significance of disease association for all three RAPTOR SNPs, with rs2019154 yielding a $p$ value of 0.008 .

- Hitherto, analysis of UK samples has failed to replicate linkage to the PSORS2 locus. The present findings emphasise the power afforded by association studies of complex trait susceptibility intervals.

TRANSMIT 2.5 software $^{11}$ to examine the transmission rates of marker alleles.

\section{RESULTS AND DISCUSSION}

Association analysis results are summarised in table 1 . We first examined the entire dataset and found that rs2019154 (distal peak) was the only marker supporting evidence for association $(p=0.027)$. To reduce the heterogeneity of our cohort, we subsequently restricted the analysis to the 116 trios that had been sampled from extended pedigrees, thus defining a subset of patients with a well documented family history of psoriasis. This stratification did not modify the outcome of the proximal peak genetic analysis. However, the significance of disease association increased for all three SNPs mapping to RAPTOR intron 3, with rs2019154 yielding the lowest $p$ value $(p=0.008)$ (table 1$)$.

Abbreviation: SNP, single nucleotide polymorphism 
Table 1 TRANSMIT output for the analysed PSORS2 SNPs

\begin{tabular}{lll}
\hline Marker & $\begin{array}{l}\text { All trios } \\
(\mathbf{n}=\mathbf{2 3 3})\end{array}$ & $\begin{array}{l}\text { Trios with positive } \\
\text { family history }(\mathbf{n}=116)\end{array}$ \\
\hline Proximal peak & & \\
SNP8 (rs7420) & $\mathrm{p}=0.44$ & $\mathrm{p}=0.53$ \\
SNP9 (rs734232) & $\mathrm{p}=0.65$ & $\mathrm{p}=0.45$ \\
SNP11 (rs895691) & $\mathrm{p}=0.21$ & $\mathrm{p}=0.24$ \\
SNP12 (rs12797) & $\mathrm{p}=0.13$ & $\mathrm{p}=0.20$ \\
SNP15 (rs2305214) & $\mathrm{p}=0.43$ & $\mathrm{p}=0.50$ \\
Distal peak & & \\
rs1564864 & $p=0.06$ & $p=0.031$ \\
rs2019154 & $p=0.027$ & $p=0.008$ \\
rs869190 & $p=0.08$ & $p=0.05$ \\
\hline
\end{tabular}

The failure to detect association with SNPs from the SLC9ARl/NAT9 genomic segment is in keeping with the low relative risk that is conferred by these variants ${ }^{9}$ and suggests that a larger dataset might be needed to replicate the significance values reported by Helms et al. ${ }^{9}$ Conversely, our observation of increased RAPTOR association in the familial subset of our sample argues for strict selection criteria in patient recruitment. In this context, it is of interest that the location of the RAPTOR gene closely matches that of the original PSORS2 interval, as defined by parametric linkage analysis of multigeneration pedigrees. ${ }^{3}$ This suggests that RAPTOR SNPs might be specifically implicated in familial psoriasis, for example by acting as a modifier of major susceptibility loci.

The observation of significant association at the RAPTOR locus is in contrast with our previous failure to detect linkage with the PSORS2 interval. ${ }^{78}$ This discrepancy could be accounted for by several factors, including the informativity and spacing of the microsatellites used in our genome scans. Moreover, the psoriasis associated alleles from this region are found on multiple haplotypes in families and would be likely to have a small effect upon disease susceptibility. Mathematical modelling has clearly shown that association studies offer a greater power to detect minor susceptibility loci, compared with linkage analysis. ${ }^{12}$ Our findings demonstrate this point on experimental data, emphasising the insight afforded by association studies in the characterisation of genomic intervals linked to complex traits.

\section{ACKNOWLEDGEMENTS}

The authors wish to thank all the psoriasis families whose participation made this project possible. We also wish to thank Isla Toal, Shenghui Duan, Patty Taillon Miller, and Jason Robarge for technical assistance.

\section{Authors' affiliations}

F Capon, C D Veal, R C Trembath, Division of Medical Genetics, University of Leicester, Leicester, UK
F Capon, Division of Human Genetics, "Tor Vergata" University of Rome, Italy

C Helms, A M Bowcock, Department of Genetics, Washington University School of Medicine, St Louis, MO, USA

D Tillman, A D Burden, Department of Dermatology, Western Infirmary, Glasgow, UK

J N Barker, St John's Institute of Dermatology, King's College, London, UK

A M Bowcock, Departments of Pediatrics and Division of Dermatology, Department of Medicine, Washington University School of Medicine, St. Lovis, MO, USA

This research was supported by a Wellcome Trust Grant to RCT and JNB (no 056713/Z/99/Z) a Medical Research Council UK Cooperative Group Grant and funding (to AMB) from the National Institutes of Health (grant no AR049049) and the National Psoriasis Foundation. FC is a recipient of a Wellcome Trust Travelling Research Fellowship.

Conflicts of interest: none declared.

Correspondence to: Prof. R Trembath, Division of Medical Genetics, Department of Genetics and Medicine, Adrian Building, University Road, Leicester LE1 7RH, UK; rtrembat@hgmp.mrc.ac.uk

Received 7 January 2004

Accepted for publication 30 January 2004

\section{REFERENCES}

1 Barker JN. Pathogenesis of psoriasis. J Dermatol 1998;25:778-81.

2 Capon F, Munro M, Barker J, Trembath R. Searching for the major histocompatibility complex psoriasis susceptibility gene. J Invest Dermatol 2002; 1 18:745-51.

3 Tomfohrde J, Silverman A, Barnes R, Fernandez-Vina MA, Young M, Lory D, Morris L, Wuepper KD, Stastny P, Menter A. Gene for familial psoriasis susceptibility mapped to the distal end of human chromosome 17q. Science 1994; 264: $1141-5$.

4 Nair RP, Henseler T, Jenisch S, Stuart P, Bichakjian CK, Lenk W, Westphal E, Guo SW, Christophers E, Voorhees JJ, Elder JT. Evidence for two psoriasis susceptibility loci (HLA and 17q) and two novel candidate regions (16q and 20p) by genome-wide scan. Hum Mol Genet 1997;6:1349-56.

5 Enlund F, Samuelsson L, Enerback C, Inerot A, Wahlstrom J, Yhr M, Torinsson A, Martinsson T, Swanbeck G. Analysis of three suggested psoriasis susceptibility loci in a large Swedish set of families: confirmation of linkage to chromosome $6 p$ (HLA region), and to $17 q$, but not to $4 q$. Hum Hered 1999;49:2-8

6 Zheng J, Jin S, Shi R. Confirmation of PSORS psoriasis susceptibility loci in a Chinese population. Arch Dermatol Res 2003;295:14-8.

7 Trembath RC, Clough RL, Rosbotham JL, Jones AB, Camp RD, Frodsham A, Browne J, Barber R, Terwilliger J, Lathrop GM, Barker JN. Identification of a major susceptibility locus on chromosome $6 p$ and evidence for further disease loci revealed by a two stage genome-wide search in psoriasis. Hum Mol Genet 1997:6:813-20.

8 Veal CD, Clough RL, Barber RC, Mason S, Tillman D, Ferry B, Jones AB, Ameen M, Balendran N, Powis SH, Burden AD, Barker JN, Trembath RC. Identification of a novel psoriasis susceptibility locus at $1 p$ and evidence of epistasis between PSORS1 and candidate loci. J Med Genet 2001;38:7-13.

9 Helms C, Cao L, Krueger JG, Wijsman EM, Chamian F, Gordon D, Heffernan M, Daw JA, Robarge J, Ott J, Kwok PY, Menter A, Bowcock AM. A putative RUNXI binding site variant between SLC9A3R1 and NAT9 is associated with susceptibility to psoriasis. Nat Genet 2003;35:349-56.

10 Speckman RA, Wright Daw JA, Helms C, Duan S, Cao L, Taillon-Miller P, Kwok PY, Menter A, Bowcock AM. Novel immunoglobulin superfamily gene cluster, mapping to a region of human chromosome 17q25, linked to psoriasis susceptibility. Hum Genet 2003;112:34-41.

11 Clayton D. A generalization of the transmission/disequilibrium test for uncertain-haplotype transmission. Am J Hum Genet 1999;65:1170-7.

12 Risch NJ. Searching for genetic determinants in the new millennium. Nature $2000 \cdot 405 \cdot 847-56$ 